\title{
Utility of subtelomeric fluorescent DNA probes for detection of chromosome anomalies in 425 patients
}

Syed M. Jalal, PhD ${ }^{1}$, Aaron R. Harwood ${ }^{1}$, Gurbax S. Sekhon, PhD ${ }^{3}$, Cindy Pham Lorentz, MS ${ }^{1}$, Rhett P. Ketterling, $M D^{1}$, Dusica Babovic-Vuksanovic, $M D^{2}$, Reid G. Meyer ${ }^{1}$, Regina Ensenauer, $M D^{2}$, Marvin H. Anderson, $\mathrm{Jr}^{1}$, and Virginia V. Michels, $M D^{2}$

\begin{abstract}
Purpose: A complete set of subtelomeric fluorescent DNA probes, except the acrocentric p-arms, was developed in 1996, was optimized in 1998, and is commercially available. These and other fluorescence in situ hybridization (FISH) probes have been used to detect anomalies of the subtelomere regions among groups of patients with idiopathic mental retardation (MR), developmental delay (DD), and/or nonspecific dysmorphic features (NDF), and individuals with multiple miscarriages (MM) who were karyotypically normal by standard G-banding techniques. Methods: A total of 425 patients were analyzed, of whom 372 had idiopathic MR/DD/NDF and 53 were involved in MM. An effort was made to select individuals for this study who were either normal karyotypically or who had subtle chromosomal anomalies that were inconclusive by banded chromosome analysis, although this was not always possible. Results: Anomalies involving the subtelomere regions were detected at a frequency of $6.8 \%$ in the MR/DD/NDF group. The cryptic or subtle anomalies are estimated to be about 3.4\%. It was necessary to use M-FISH, chromosome, and locus specific FISH probes to clarify some of the abnormalities. No abnormalities were detected in the MM group. Deletion variants were present for 2qter, 7pter, and Xpter/Ypter subtelomeric regions ranging from $<1$ to $9.6 \%$. Conclusions: The subtelomeric FISH probes are instrumental in the detection of subtelomeric anomalies in a significant proportion, although no more than $50 \%$ are subtle, of patients with idiopathic MR/DD/NDF. In some cases, however, it was necessary to use other FISH probes to clarify the nature of these abnormalities. No subtelomeric abnormalities were detected in our group of $53 \mathrm{MM}$ patients, suggesting a relatively low frequency of occurrence in this patient population. Genet Med 2003:5(1):28-34.
\end{abstract}

Key Words: subtelomere, FISH probes, cryptic anomalies

Since the late 1980s, telomere caps are known to contain (TTAGGG) repeat sequences ranging from 2 to $20 \mathrm{~Kb} .{ }^{1}$ It is now known that telomere associated repeat sequences (TAR) extend to 100 to $300 \mathrm{~Kb}$ from the terminal repeat sequences. ${ }^{2,3}$ Chromosome specific unique sequences are located centromeric to the TAR region. A complete set of subtelomere specific probes for each chromosome except the acrocentric parms was developed by the National Institutes of Health $(\mathrm{NIH}) .{ }^{4}$ Some of these earlier probes appear to be from the highly polymorphic TAR region. These probes were optimized to increase the target size to 100 to $200 \mathrm{~Kb}$ and were derived primarily from the unique DNA sequences located within 500 $\mathrm{Kb}$ of the end of each chromosome arm. ${ }^{5}$ Martin et al. ${ }^{6}$ have

From the ${ }^{1}$ Cytogenetics Laboratory, Department of Laboratory Medicine and Pathology, Mayo Clinic and Mayo Foundation, Rochester, Minnesota; ${ }^{2}$ Department of Medical Genetics, Mayo Clinic and Mayo Foundation, Rochester, Minnesota; and ${ }^{3}$ University of Wisconsin, Waisman Center, Madison, Wisconsin.

Syed M. Jalal, PhD, Cytogenetics Laboratory, Department of Laboratory Medicine and Pathology, Mayo Clinic, 200 First Street SW, Rochester, MN 55905.

Received: August 8, 2002.

Accepted: October 28, 2002.

DOI: 10.1097/01.GIM.0000046363.02928.AE identified nonunique clones from this region that may have evolved as ancestral duplications and may well be the cause of cross-hybridizations of telomere specific probes. On the basis of this information, subtelomere fluorescence in situ hybridization (FISH) probes were commercially developed for each end of chromosome arms except the acrocentric p-arms. Because of the high gene concentration in the subtelomere regions, ${ }^{7}$ there is an intense interest in investigating cryptic abnormalities $^{8}$ of these regions, especially in patients with nonspecific dysmorphic features (NDF), developmental delay (DD), and/or cryptic mental retardation (MR), especially when the standard karyotype is normal.

We investigated the occurrence of subtle subtelomeric anomalies by use of commercial subtelomeric FISH probes in 372 cases of MR/DD/NDF patients and 53 individuals involved in multiple miscarriages (MM).

\section{MATERIALS AND METHODS}

\section{Strategy}

A total of $372 \mathrm{MR} / \mathrm{DD} / \mathrm{NDF}$ patients and $53 \mathrm{MM}$ individuals were analyzed for subtelomeric abnormalities primarily by the commercially available subtelomere specific FISH probes. For 
those patients evaluated at the Mayo Clinic (about 20\%), the subtelomere analysis was performed if the karyotype was normal at the 550 band stage or the G-banded analysis was ambiguous. For those patients not clinically evaluated at the Mayo Clinic, the karyotype was performed in this laboratory or elsewhere, and the results were either normal or ambiguous; the majority of these patients were karyotyped elsewhere. For the MM group, the G-banded karyotype from stimulated whole blood culture (at 550 band stage or higher) was analyzed before the subtelomere FISH investigation. Every effort was made to confirm or to provide further details whenever an abnormality or a new polymorphism was observed in a patient by analysis of the parents and by use of the probes from the other company. In a few complex cases, M-FISH, ${ }^{9}$ whole chromosome painting probes, and locus specific probes were used to clarify the abnormality.

\section{Commercially available telomere specific probes}

The protocol for Cytocell (Oxfordshire, United Kingdom) was as follows: Metaphase cells in fixative suspension were carefully dropped alternately onto the 24 squares of the template slide. Concentrated cell suspensions were evaluated with phase microscopy for adequacy of metaphases (large numbers) and were placed on the slide in a Thermatron (Methuen, MA) drying chamber ${ }^{10}$ for proper spreading and morphology. The template slide and the probe device were prepared for codenaturation and hybridization according to Cytocell instructions.

The protocol for Vysis subtelomere probes (Downers Grove, IL) involved the following: metaphase cells from a concentrated fixative cell suspension were dropped in a similar fashion as described above in a Thermatron chamber on three slides in five defined areas on each slide. The 15 probe mixtures provided by the manufacturer were used according to the instructions provided.

Following the hybridization with Cytocell and Vysis probes, the slides were washed in $0.4 \mathrm{xSSC}$ for 2 minutes (after removal of the probe device in Cytocell protocol and coverslip in the Vysis protocol), rinsed in $0.1 \% \mathrm{NP} 40 / 2 \times \mathrm{xSC}$, and counterstained with 4',6'-diamidine-2-phenylindole dihydrochloride (DAPI). At least three metaphases were analyzed for each of the 22 autosomes and $\mathrm{X}$ and $\mathrm{Y}$ chromosomes using fluorescence microscopy. Digitized images were stored for printing or future use.

We started using Cytocell probes in 1999, but since 2000 we have primarily been using Vysis probes. Our technical experience with the probes and strategies of the two companies has been previously described. ${ }^{10}$

\section{RESULTS}

\section{Representative cases}

Case 14

A 2-week-old male was referred to rule out subtelomere anomaly. The karyotype appeared normal at 550 band stage. The results of complete subtelomere probe set analysis indi- cated a normal pattern for chromosome pairs 13 and 1, but 13qter signal was also present at 1qter, indicating an insertional duplication of 13qter as a cryptic anomaly. Both chromosomes 1 and 13 homologues showed complete hybridization with wcp1 and wcp13 probes (Fig. 1, Case 14). Parental blood samples have been requested for analysis.

\section{Case 10}

A 12-year-old female was referred for DD (Table 1). The chromosome 10qter appeared abnormal by G-banded analysis, but whether it was a deletion or derivative chromosome could not be determined. Use of the subtelomere probe set confirmed the anomaly to be a terminal deletion (Fig. 1, Case 10). The parents were not available for chromosome or subtelomere analysis.

\section{Case 18}

A 14-year-old female was referred for DD (Table 2). By using G-band by trypsin using Leishman Giemsa stain (GTL) banded chromosome analysis, an abnormality was detected as add (4)(q35). The use of the subtelomere probe set determined that the abnormal chromosome 4 was $\operatorname{der}(4) \mathrm{t}(2 ; 4)$ (pter;qter). Thus the abnormal chromosome 4 had a deletion of 4 qter and duplication of 2pter (Fig. 1, Case 18). Parents were unavailable for chromosome or subtelomere analysis.

\section{Case 15}

A newborn was referred to rule out a balanced familial translocation of $\mathrm{t}(2 ; 11)(\mathrm{q} 37.3 ; \mathrm{q} 25)$ (Table 2). Telomere specific probe set indicated a deletion of 2qter, as expected for translocation to 11qter, but the pattern of chromosome 11pter and 11qter was unexpectedly normal. Use of M-FISH and wcp11 confirmed that the infant had a complex balanced reciprocal exchange between the q-arm subtelomeric regions of chromosomes 2 and 11 (Fig. 1, Case 15).

\section{Cases $19 A$ and $19 B$}

A 3-month-old male with multiple dysmorphic features, who was a product of assisted reproduction, was referred to medical genetics for evaluation (Table 2). The infant and an older sister shared similar multiple dysmorphic features, and both had DD. The male infant also had ambiguous genitalia due to known chimerism (about 12\% cells were 46,XX) that was confirmed by our laboratory. We also confirmed a known de novo translocation $\mathrm{t}(4 ; 5)$ ( $\mathrm{q} 31.1 ; \mathrm{q} 14)$ that he carried. By use of the subtelomere specific probes, it was discovered that the abnormal chromosome 5 had a deletion of 5 pter and duplication of 17 pter that was not detected by banded chromosome or M-FISH analysis. Thus the abnormal chromosome 5 was $\operatorname{der}(5) t(5 ; 17)$ (pter;pter) (Fig. 2, Case 19A). The older sister, who was karyotypically normal by G-banding, had the same abnormal der(5). The father was shown to have a balanced translocation $\mathrm{t}(5 ; 17)$ (pter;pter) (Fig. 2). 


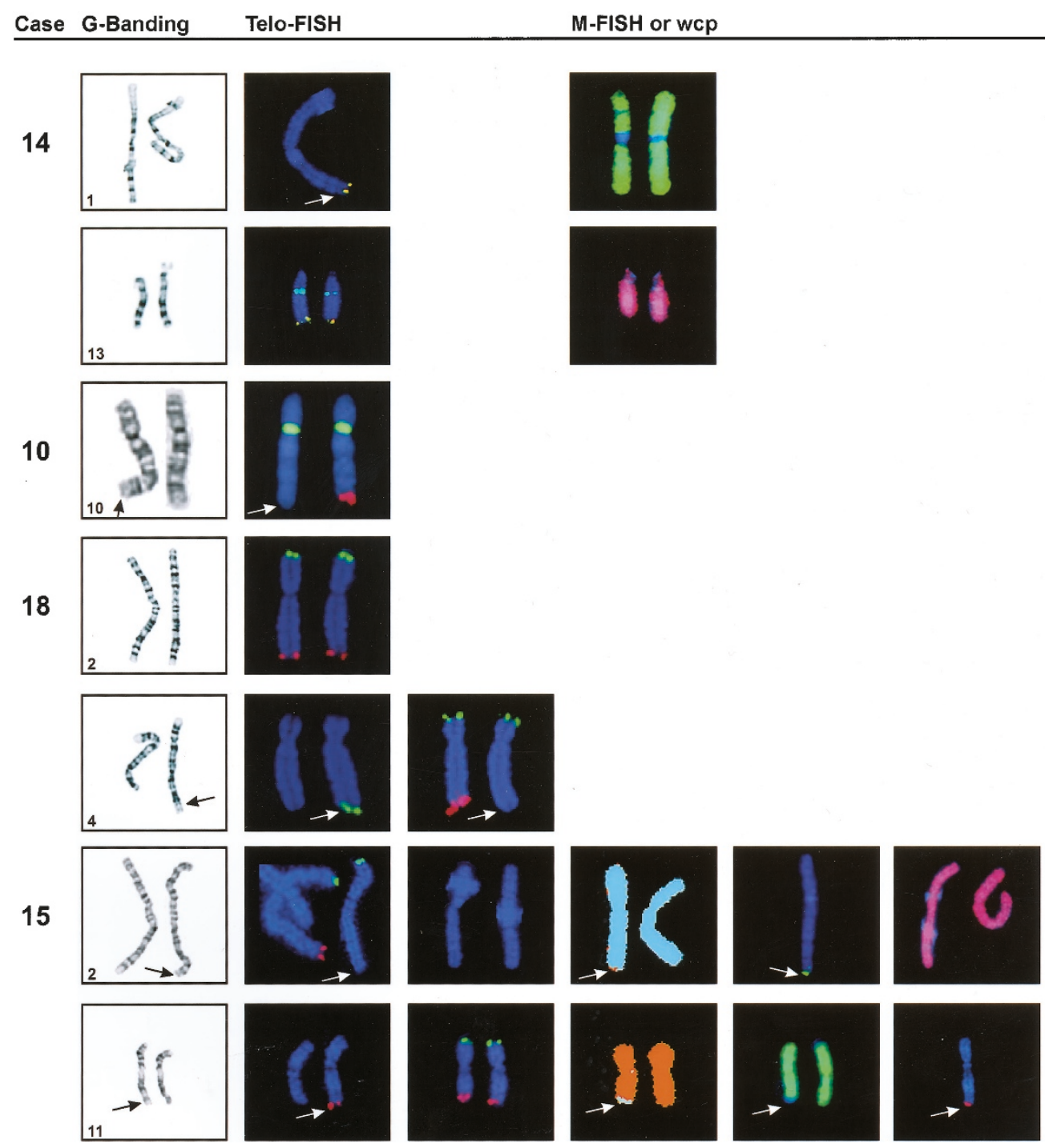

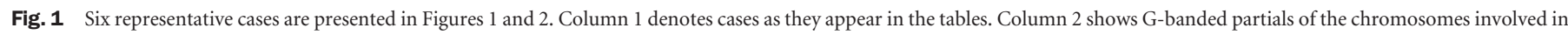

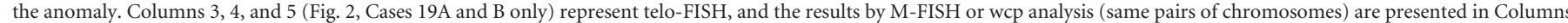

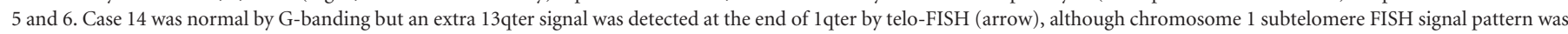

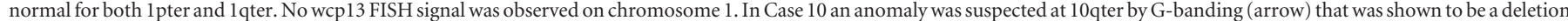

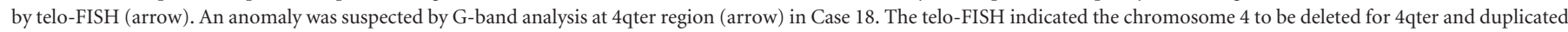

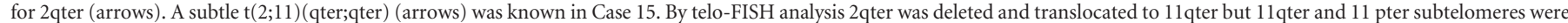
present on both homologous chromosomes (arrows). Use of M-FISH, wcp2, and wcp11 revealed an exchange between chromosomes 2 and 11 (arrows).

\section{Anomalies detected by subtelomeric probes}

The patients from the MR/DD/NDF group with deletions or duplication detected by subtelomere probes are presented in Table 1. Some of the highlights are as follows: Of the two cases with 1pter deletion, p58 or p73 loci at 1p36.2 was deleted in Case 2 but was not deleted in Case 1. The 4pter deletion (Case 5) was reported to us as normal karyotypically at 500 band stage. In addition to the 4 pter deletion detected by subtelomere probes, the Wolf-Hirschhorn critical region was also deleted. The 8pter deletion (Case 9) was familial, and the deletion was also demonstrated in the maternal grandmother, mother, and a brother. Carriers of the abnormality had DD, speech delay, and significant IQ deficit. Through banded chromosome analysis, it seemed that the deletion may be associated with a subtle duplication (the banded analysis was performed by another laboratory). The 14qter deletion (Case 11) was a product of $\mathrm{t}(14 ; 21)(\mathrm{q} 32.3 ; \mathrm{q} 11.2),-21$, so that the $\operatorname{der}(14)$ was missing $14 q$ ter, and almost the entire $21 \mathrm{q}$ was translocated to the der(14), although some loss from proximal 21q-arm cannot be ruled out. The 22qter deletion (Case 12) did not involve the deletion of arylsulfatase (ARSA). Excluding the family with 8pter deletion, we analyzed one or both parents in the three other cases. In each case, parental analysis was normal.

There were two cases of duplication. One involved a tandem duplication, and the other was insertional duplication. The case involving 8qter (Table 1, Case 13) had a tandem cryptic duplication involving subtelomeric probes. The duplication was not visible by G-banding. Unfortunately, we have very 
Table 1

Detected deletions/duplications of the subtelomere region

\begin{tabular}{|c|c|c|c|c|c|}
\hline Patient & Age/gender & Abnormality & Reason for referral & Parents/available family members & $\begin{array}{l}\text { Family } \\
\text { results }\end{array}$ \\
\hline 1 & $3 \mathrm{Y} / \mathrm{M}$ & $\operatorname{del}(1)($ pter $)$ & r/o deletion/rearrangement & Mother & Normal \\
\hline 2 & $2 \mathrm{Y} / \mathrm{F}$ & $\operatorname{del}(1)($ pter $)$ & DD, nonspecific MR and dysmorphism & None & \\
\hline 3 & $3 \mathrm{Y} / \mathrm{F}$ & $\operatorname{del}(1)$ (qter) & Multiple congenital anomalies & Mother, father & $\begin{array}{l}\text { Normal } \\
\text { Normal }\end{array}$ \\
\hline 4 & $2 \mathrm{Y} / \mathrm{F}$ & $\operatorname{del}(2)($ pter $)$ & r/o deletion or rearrangement & None & \\
\hline 5 & $6 \mathrm{Y} / \mathrm{F}$ & $\operatorname{del}(4)($ pter $)$ & DD, hypertelorism & None & \\
\hline \multirow[t]{2}{*}{6} & $6 \mathrm{D} / \mathrm{F}$ & $\operatorname{del}(4)$ (qter) & $\begin{array}{l}\text { Aortic arch and skeletal anomaly, pulmonary } \\
\text { and esophageal atresia, TE fistula }\end{array}$ & Mother & Normal \\
\hline & & & & Father & Normal \\
\hline 7 & $7 \mathrm{Y} / \mathrm{M}$ & $\operatorname{del}(8)($ pter $)$ & $\begin{array}{l}\text { r/o chromosome abnormality karyotype } \\
\text { del }(8)(\mathrm{p} 23.1)\end{array}$ & None & \\
\hline 8 & $2 \mathrm{Y} / \mathrm{M}$ & $\operatorname{del}(8)($ pter $)$ & $\begin{array}{l}\text { Macrocephaly, hydrocephaly, dysmorphic } \\
\text { features }\end{array}$ & None & \\
\hline \multirow[t]{2}{*}{$9^{a}$} & $11 \mathrm{Y} / \mathrm{M}$ & $\operatorname{del}(8)($ pter $)$ & DD, dyslexia, significant IQ deficit & $\begin{array}{l}\text { Brother, mother, maternal } \\
\text { grandmother, father, grandfather }\end{array}$ & \\
\hline & & & & & Normal \\
\hline 10 & $12 \mathrm{Y} / \mathrm{F}$ & $\operatorname{del}(10)($ qter $)$ & $\mathrm{DD}$ & None & \\
\hline $11^{b}$ & $7 \mathrm{Y} / \mathrm{F}$ & $\operatorname{del}(14)$ (qter) & DD, microcephaly, minor anomalies & None & \\
\hline 12 & $2 \mathrm{Y} / \mathrm{F}$ & $\operatorname{del}(22)$ (qter) & $\mathrm{DD}$ & None & \\
\hline 13 & $10 \mathrm{Y} / \mathrm{M}$ & dup(8qter) & r/o subtelomere abnormality & None & \\
\hline 14 & $2 \mathrm{~W} / \mathrm{M}$ & ins dup(13qter) & r/o subtelomere abnormality & Requested & \\
\hline
\end{tabular}

r/o, rule out; DD, developmental delay; MR, mental retardation; TE, tracheo-esophageal.

${ }^{a}$ Involves duplication of 8p23.1-8p23.3 as well by high-resolution G-banding.

${ }^{b}$ Standard karyotype was 45,XX,der(14)t(14;21)(q32.3;q11.2), -21 .

limited information about the associated phenotype, which is true for many of these cases (Table 1). A subtelomere study has been recommended for the parents. The second case was a cryptic insertional duplication involving 13qter subtelomere region to 1qter. This was undetectable by either M-FISH or painting probes.

The cases with either the derivative chromosomes or balanced translocations from the MR/DD/NDF population are presented in Table 2. Some of the highlights are as follows: In six cases, parents were available for analysis. Subtelomere analyses revealed that four of the patients carrying a derivative chromosome or a balanced translocation were familial. In two cases, the derivative chromosome appeared to arise de novo. Case 15 has been described earlier in this section. In this reciprocal translocation, the qter of the der(11) hybridized to both 11qter and 2qter while the der(2) hybridized to neither probe. Subtelomere and M-FISH results indicate a complex reciprocal exchange. Case 19A and 19B were described earlier in this section. Both children have the same der(5)t(5;17)(pter;pter) that could not be detected by banded chromosome analysis or M-FISH. The der(22) of Case 23 resulted from a t 1 13;22)(p12; q13.3). The der(22) had a deletion of 22qter and the ARSA locus at $22 \mathrm{q} 13.3$, which were proximal to the translocated segment. Thirty cases were abnormal from a total of 425 cases studied for a frequency of $6.8 \%$. However, the frequency of cryptic or subtle anomalies is much lower (about 50\%) if only those cases are included that are ascertained by the subtelomere test and not those suspected by G-banding.

\section{Polymorphism and cross-hybridizations}

Cytocell lists potential cross-hybridizations of the subtelomeric probes on 10 chromosomes. Vysis does not list specific cross-hybridizations with their subtelomere probe set, but, depending on stringency conditions, we have observed $3 \mathrm{q}, 8 \mathrm{p}, 8 \mathrm{q}$, and $9 \mathrm{q}$ to cross-hybridize. ${ }^{11}$ In addition, $4 \mathrm{p}$ was also observed recently to cross-hybridize to $17 \mathrm{p}$ in our laboratory. Fortunately, the cross-hybridization signals were often smaller and not present in every cell.

We have previously reported a 2qter deletion polymorphism involving the Cytocell subtelomere probe set. ${ }^{12}$ In the first such case, a normal father had the deletion variant and the Vysis probe results were normal. In only two cases $(<1 \%)$, a $7 \mathrm{p}$ deletion variant was observed using both the Cytocell and $\mathrm{Vy}$ sis probes. In each case, normal parents had the deletion of the 7 pter probe. Three cases of Xp or Yp deletion variants have been observed, but the true nature of this potential variant has not been confirmed in two cases because parents were unavailable for analysis. 
Table 2

Detected derivative chromosomes or a balanced translocation involving the subtelomere regions

\begin{tabular}{|c|c|c|c|c|c|}
\hline Patient & Age/gender & Abnormality & Reason for referral & $\begin{array}{l}\text { Available family } \\
\text { members }\end{array}$ & Family results \\
\hline 15 & Newborn M & $\mathrm{t}(2 ; 11)(\mathrm{q} 37.3 ; \mathrm{q} 25)$ & Familial translocation & Father, mother & $\begin{array}{l}\mathrm{t}(2 ; 11)(\mathrm{q} 37.3 \mathrm{q} 25) \\
\text { Normal }\end{array}$ \\
\hline 16 & $3 \mathrm{Y} / \mathrm{F}$ & $\operatorname{der}(3) t(3 ; 17)$ (pter;qter) & $\mathrm{DD}$ & Father, mother & $\begin{array}{l}\mathrm{t}(3 ; 17)(\text { pter;qter }) \\
\text { Normal }\end{array}$ \\
\hline 17 & $7 \mathrm{M} / \mathrm{M}$ & $\operatorname{der}(4) \mathrm{t}(4 ; 8)($ pter;pter $)$ & Failure to thrive, MCA & Father, mother & $\begin{array}{l}\text { Normal } \\
\text { Normal }\end{array}$ \\
\hline 18 & $14 \mathrm{Y} / \mathrm{F}$ & $\operatorname{der}(4) \mathrm{t}(2 ; 4)$ (pter;qter) & $\mathrm{DD}$ & None & \\
\hline \multirow[t]{2}{*}{$19 \mathrm{~A}$} & 3Y/M brother & $\begin{array}{c}\operatorname{der}(5) \mathrm{t}(5 ; 17) \text { (pter;pter) } \\
\mathrm{t}(4 ; 5)(\mathrm{q} 31.1 ; \mathrm{q} 14)\end{array}$ & Ambiguous genitalia, DD, minor & Father & $\mathrm{t}(5 ; 17)$ (pter;pter) \\
\hline & & & Dysmorphic features, heart murmur & Mother & Normal \\
\hline 19B & 2Y/F sister & $\operatorname{der}(5) t(5 ; 17)($ pter;pter $)$ & $\mathrm{DD}$, minor dysmorphic features, heart murmur & & \\
\hline 20 & $7 \mathrm{~W} / \mathrm{M}$ & $\mathrm{t}(6 ; 17)$ (pter;qter) & r/o cryptic deletion $\mathrm{t}(6 ; 17)(\mathrm{q} 27 ; \mathrm{q} 25)$ & None & \\
\hline 21 & $5 \mathrm{~W} / \mathrm{F}$ & $\operatorname{der}(7) t(7 ; 20)$ (qter;qter) & r/o Down syndrome & Father, mother & $\begin{array}{l}\text { Normal } \\
\text { Normal }\end{array}$ \\
\hline 22 & $1 \mathrm{Y} / \mathrm{F}$ & $\operatorname{der}(20) \mathrm{t}(4 ; 20)$ (pter;pter) & r/o subtelomeric deletion or rearrangement & None & \\
\hline 23 & $6 \mathrm{Y} / \mathrm{M}$ & $\operatorname{der}(22) t(13 ; 22)($ pter;qter $)$ & r/o subtelomere anomaly & Father, mother & $\begin{array}{l}\operatorname{der}(22) t(13 ; 22) \text { (pter;qter) } \\
\text { Normal }\end{array}$ \\
\hline
\end{tabular}

r/o, rule out; DD, developmental delay; MCA, multiple congenital anomalies.

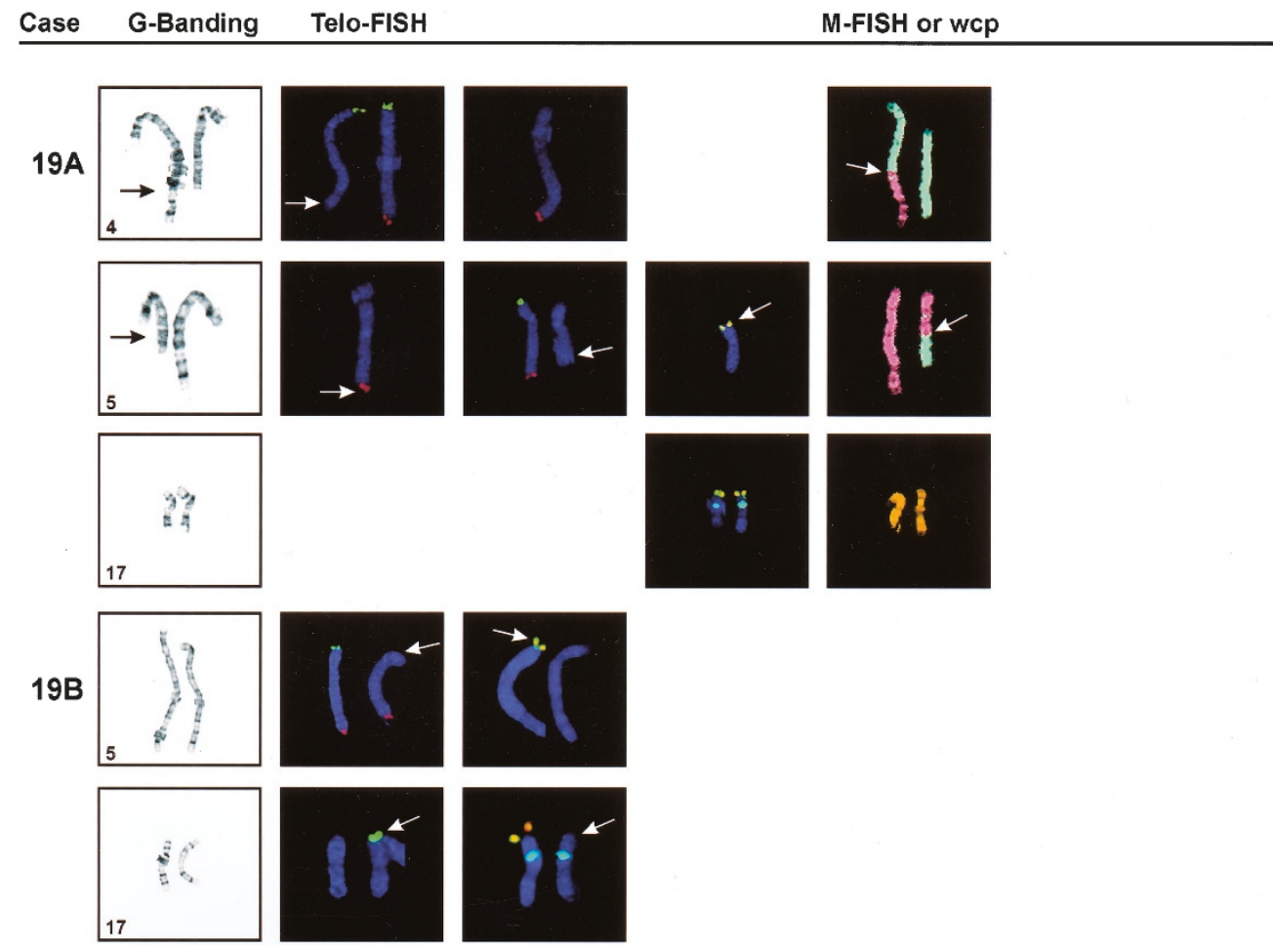

Fig. 2 Figure 2 columns are organized like Figure 1 columns. In Case 19A, a balanced translocation between $4 \mathrm{q}$ and $5 \mathrm{q}$ was identified (arrows) by G-banding. Telo-FISH detected the $\operatorname{der}(5) \mathrm{t}(4 ; 5)$ to have a deletion of 5 pter and was replaced with $17 \mathrm{pter}$ (arrows). The 5 pter anomaly was not detected by G-banding, M-FISH, or wcps. The father (19B) carried the balanced subtle translocation involving 5pter and 17pter (arrows).

\section{Multiple miscarriages group}

We decided to investigate subtelomeric abnormalities in the MM group because we were not aware of any other report. The frequency of subtelomere abnormalities in this group of 53 individuals referred for a history of MM was $0 \%$. Each patient was karyotypically normal at 500 or higher band resolution. 
Deletion variant involving 2qter was detected at a relatively high frequency of $9.4 \%$ in this group. de Vries et al. ${ }^{13}$ observed that history of miscarriage in their control group and in subtelomeric cases were statistically not different.

\section{DISCUSSION}

Subtelomeric FISH probes are effective in the determination of cryptic deletions, duplications (Fig. 1; Cases 10 and 14), or derivative chromosomes resulting from subtle translocations of the telomere region (Figs. 1 and 2; Cases 15 and 19) as illustrated by these cases. These probes are also very helpful in sorting out cryptic derivative chromosomes in the presence of other abnormalities where the cryptic anomaly was missed by G-banded chromosome analysis and M-FISH. However, in some cases of complex anomalies, the sole use of the subtelomere probes was inadequate. In one of the cases described (Fig. 1, Case 15), the subtle reciprocal exchange involving the terminal bands (2q37.3 and 11q25) was detected by high-resolution karyotype. This translocation was familial, and it was known that der(11) infants appeared normal at birth and later developed autism and other minor anomalies, while der(2) carriers were more dysmorphic at birth. We investigated a phenotypically normal appearing newborn male by subtelomeric probe set. The 2qter probe hybridized with 11qter in one of the chromosomes 11 as expected, but the chromosome 11 subtelomere probes hybridized normally to chromosome 11 . The reciprocal exchange between 2 and 11 was large enough to be detected by M-FISH, wcp11, and high-resolution banding (Fig. 1, Case 15). On the basis of the apparent "discordant" results between subtelomeric probes and M-FISH, wcps, and G-banding, we believe that this case results from two breaks (one in the TAR region) on chromosome 2 that includes the subtelomeric probe and an interstitial deletion-translocation proximal to the $11 \mathrm{q}$ subtelomere probe. When these fragments are exchanged, the 11qter ends up with both the 2qter subtelomere and 11qter subtelomeric probes, and 2qter has no DNA sequences homologous to the FISH subtelomere probe. The other possibility is that there were distal and proximal breakpoints followed by an exchange involving the probes of 2qter and 11qter, but it was detectable only on 11qter. The subtelomeric probe data would, therefore, be inconclusive or could be easily misinterpreted if used by themselves. Case 14 (Fig. 1) is unique in that the abnormality detected is entirely cryptic. The subtelomere probe set analysis indicated an extra 13qter signal at 1qter while chromosome 1 subtelomere hybridization signal pattern was normal at both pter and qter ends. G-banded analysis at 550 band stage and results of wcp 1, wcp 13 were all normal. It was therefore interpreted to be a case of cryptic insertional duplication involving 13qter subtelomere. Study of other family members has been requested.

Knight et al. ${ }^{14}$ published the results of the first comprehensive study of subtle anomalies of the subtelomere regions by use of subtelomere FISH probes in a group of children with idiopathic MR. They discovered $7.4 \%$ abnormalities in the group with moderate to severe MR ( $<50$ IQ scores). At 550 band stage, all of these cases were reported as normal, although, after the FISH-based analysis results were known, about $20 \%$ of the abnormalities were detectable by high-resolution banding. Recently, other researchers have reported similar rates of abnormalities in this patient population (4.0-9.0\%). ${ }^{15-17}$ An editorial ${ }^{17}$ accompanying these three studies concludes that segmental aneusomy of the subtelomeric region accounts for $6 \%$ average frequency in MR/NDF populations. Our result is consistent with these reports at about $6.8 \%$, although subtle or cryptic anomalies were no more than $3.4 \%$. In an effort to improve the chances for detection of subtelomeric abnormalities, de Vries et al. ${ }^{13}$ developed a checklist of phenotypic features on the basis of 29 patients with a known subtelomeric abnormality. This may help in the preselection process, especially if the karyotype is normal at 500 band resolution or higher.

Biesecker ${ }^{8}$ observed that the detection of subtelomeric anomalies by high-resolution chromosome analysis has been reported to range from $0 \%$ to near $100 \%$. Strategically, one must begin the cytogenetic investigation by banded chromosome analysis at 500 band stage or higher. We have come to rely on an array of FISH probes for detection of relatively subtle anomalies to complement banded chromosome analysis. The subtelomeric probes helped for segmental aneusomy detection of the terminal regions not only when the banded karyotype was normal, but they were also instrumental in clarification of anomalies when they are suspected by banded chromosome analysis.

The list of polymorphic variants and cross-hybridizations of the subtelomeric FISH probes is growing. ${ }^{11}$ We previously reported the 2qter deletion variant/polymorphism to occur with a frequency of $9.4 \% .{ }^{12}$ Fan et al. ${ }^{18}$ similarly report the frequency of this variant to be at $6 \%$ in a population of idiopathic MR patients. The 2qter variant has been reported to be about $5 \%$ with the commercial probes that are larger in size compared with earlier versions. ${ }^{19}$ The other rare deletion variants thus far described are 7pter and Xpter/Ypter. Some of the polymorphic variants can be rare and unexpected, such as that reported for lqter. ${ }^{20}$ When such variants are encountered, parents need to be carefully evaluated by the subtelomeric probe set and by physical examination to document their phenotype. If a parent shares some of the same phenotypic abnormalities, it is possible that deletion size may be larger in the affected child. This hypothesis can be confirmed by use of labeled bacterial artificial chromosomes or molecular analysis of dinucleotide repeats in that region. Conversely, if a phenotypically normal parent has the same deletion as the child, this would strongly suggest that the deletion may be a variant, although segmental imprinting effects must still be considered.

\section{CONCLUSION}

Commercially available sets of subtelomeric FISH probes are yielding a wealth of data for segmental aneusomy of the subtelomeric region at a significant proportion $(\geq 6 \%)$ of patients with idiopathic MR, DD, or NDF. However, no more than $3.4 \%$ had a subtle or cryptic abnormality. Use of the sub- 
telomere FISH probes was also very helpful in an accurate description of the abnormalities suspected by G-banding in a time-efficient manner. Care needs to be taken to complement this investigation with other FISH probes or molecular techniques when complex abnormalities are suspected. Parents should be studied whenever a subtelomeric aneusomy is identified, and these families should be seen by a medical genetics specialist for appropriate evaluation and counseling. It is helpful in the interpretation of data to continue to accumulate the information on each subtelomeric segmental aneusomy for phenotypic abnormalities, as well as polymorphic variants and cross-hybridizations of each subtelomere specific probe.

\section{References}

1. Moyzis RK, Buckingham JM, Cram LS, Dani M, Deaven LL, Jones MD et al. A highly conserved repetitive DNA sequence, (TTAGGG)n, present at the telomeres of human chromosomes. Proc Natl Acad Sci U S A 1988;85:6622-6626.

2. Brown WR, MacKinnon PJ, Villasante A, Spurr N, Buckle VJ, Dobson MJ. Structure and polymorphism of human telomere-associated DNA. Cell 1990;63:119-132.

3. Knight SJ, Flint J. Perfect endings: a review of subtelomeric probes and their use in clinical practice. J Med Genet 2000;37:401-409.

4. National Institute of Health and Institute of Molecular Medicine. A complete set of human telomeric probes and their clinical application. Nat Genet 1996;14:86-89.

5. Knight SJ, Lese CM, Precht KS, Kuc J, Ning Y, Lucas S et al. An optimized set of human telomere clones for studying telomere integrity and architecture. Am J Hum Genet 2000;67:320-323.

6. Martin CL, Wong A, Gross A, Chung J, Fantes JA, Ledbetter DH. The evolutionary origin of human subtelomeric homologies: or where the ends begin. Am J Hum Genet 2000;70:972-984.

7. Saccone S, DeSario A, Valle GD, Bernardi G. The highest gene concentrations in the human genome are in telomeric bands of metaphase chromosomes. Proc Natl Acad Sci U S A 1992;89:4913-4917.
8. Biesecker LG. The end of the beginning of chromosome ends. Am J Med Genet 2002;107:263-266.

9. Jalal SM, Law ME. Utility of multicolor fluorescent in situ hybridization in clinical cytogenetics. Genet Med 1999;1:181-186.

10. Spurbeck JL, Zinsmeister AR, Meyer KJ, Jalal SM. Dynamics of chromosome spreading. Am J Med Genet 1996;61:387-393.

11. Harwood A, Anderson M, Jalal SM. Technical comparison of two commercially available telomere specific probe set. J Assoc Genet Tech 2001;27:132-134.

12. Jalal SM, Harwood AR, Anderson MH, Sekhon GS, Ketterling RP, Michels VV. Analysis by telomere specific FISH probes of 191 karyotypically normal patients with nonspecific dysmorphic features or developmental delay, or history of multiple miscarriages. Am J Hum Genet 2001;69:333

13. de Vries BB, White SM, Knight SJ, Regan R, Homfray T, Young ID et al. Clinical studies on submicroscopic subtelomeric rearrangements: a checklist. J Med Genet 2001;38:145-150.

14. Knight SJ, Regan R, Nicod A, Horsley SW, Kearney L, Homfray T et al. Subtle chromosomal rearrangements in children with unexplained mental retardation. Lancet North Am Ed 1999;354:1676-1681.

15. Baker E, Hinton L, Callen DF, Altree M, Dobbie A, Eyre HJ et al. Study of 250 children with idiopathic mental retardation reveals nine cryptic and diverse subtelomeric chromosome anomalies. Am J Med Genet 2002;107:285-293.

16. Clarkson B, Pavenski K, Dupuis L, Kennedy S, Meyn S, Nezarati MM et al. Detecting rearrangements in children using subtelomeric FISH and SKY. Am J Med Genet 2002;107:267-274.

17. Anderlid B-M, Schoumans J, Annerén G, Sahlén S, Kyllerman M, Vujic M et al. Subtelomeric rearrangements detected in patients with idiopathic mental retardation. Am J Med Genet 2002;107:275-284.

18. Fan Y-S, Zhang Y, Speevak M, Farrell S, Jung JH, Siu VM. Detection of submicroscopic aberrations in patients with unexplained mental retardation by fluorescence in situ hybridization using multiple subtelomeric probes. Genet Med 2001;3:416421.

19. Ballif BC, Kashork CD, Shaffer LG. The promise and pitfalls of telomere regionspecific probes. Am J Hum Genet 2000;67:1356-1359.

20. Shaffer LG, Kashork CD, Bacino CA, Benke PJ. Telomere crossing. Am J Med Genet $1999 ; 87: 278-280$. 